\title{
Localisation des résidus de Baum-Bott, courbes généralisées et K-théorie (I: feuilletages dans $\mathbb{C}^{2}$ )
}

\author{
Vincent Cavalier et Daniel Lehmann
}

\begin{abstract}
Let $v$ be a holomorphic vector field in a neighborhood of a point $m_{0}$ in $\mathbb{C}^{2}$, which is a non dicritical isolated singularity. Let $f=0$ be a reduced equation of the maximal separatrix $V$ through $m_{0}, v_{f}$ the vector field $v_{f}=f_{y}^{\prime} \frac{\partial}{\partial x}-f_{x}^{\prime} \frac{\partial}{\partial y}$, and $\Sigma$ the union of separatrices and pseudo-separatrices (i.e. the set of points where $v$ and $v_{f}$ are colinear). Assuming the foliations defined by $v$ and $v_{f}$ to be distinct, we prove that the Baum-Bott residue $B B\left(c_{1}^{2}, v\right)$ of $v$ at $m_{0}$, as well as the difference $P H(v)-\mu$ between the Poincaré-Hopf index and the Milnor number of $V$ at $m_{0}$, are "localised" near the separatrices and pseudo-separatrices. (The particular case of generalized curves has already been studied in details in [CLS] and [Br]). We also interpret in K-theory the difference $P H-\mu$ as well as the GSV index of Gomez Mont-Seade-Verjovski, and we give a caracterisation of generalized curves in this framework, which will enable us to extend this concept in higher dimension.
\end{abstract}

Mathematics Subject Classification (2000). 57R20, 57R25, $19 \mathrm{E} 20$.

Keywords. Singular holomorphic foliations, generalized curves, $K$-theory.

\section{Introduction}

Soit $v=a \frac{\partial}{\partial x}+b \frac{\partial}{\partial y}$ un champ de vecteurs holomorphe, défini sur un voisinage $U$ d'un point $m_{0}$ de $\mathbb{C}^{2}$, et ayant en ce point une singularité isolée non dicritique. [On conviendra de réserver cette terminologie au cas ou aucune composante dicritique n'apparaît au cours du processus de réduction des singularités.] Soit $V$ la séparatrice maximale en ce point (i.e. la réunion de toutes les séparatrices qui passent par $m_{0}$, nécessairement en nombre fini puisque la singularité $m_{0}$ a été supposée non dicritique).

Si $f=0$ est une équation réduite de $V$, C. Camacho, A. Lins Neto et P. Sad ont cherché, dans [CLS], à comparer le feuilletage défini par $v$ avec le feuilletage $f=c^{t e}$ défini par le champ de vecteurs $v_{f}=f_{y}^{\prime} \frac{\partial}{\partial x}-f_{x}^{\prime} \frac{\partial}{\partial y}$. Ils ont appelé "courbe généralisée" les feuilletages dont la résolution par éclatements a les mêmes singularités que $v_{f}$, c'est à dire n'admet que des singularités simples à valeurs propres toutes non nulles (pas de "selle-noeud"). Ils ont montré dans ce cas, que l'indice 
$P H_{m_{0}}(v)$ de Poincaré-Hopf de $v$ est égal au nombre de Milnor $\mu$ de $V$ en $m_{0}$, et que réciproquement cette propriété caractérise les courbes généralisées parmi les singularités non dicritiques.

Dans $[\mathrm{Br}], \mathrm{M}$. Brunella a comparé plus généralement les différents indices en un point singulier non dicritique $m_{0}$. Il a montré que l'indice $G S V_{m_{0}}(v, V)$ de Gomez Mont-Seade-Verjovski de $v$ en $m_{0}$ relatif à $V$ (cf. ([GSV]) était toujours positif ou nul. Dans le cas d'une courbe généralisée et pour une séparatrice $V$ maximale, il a montré que $G S V_{m_{0}}(v, V)$ était nul, tandis que le résidu de Baum-Bott $B B_{m_{0}}\left(v, c_{1}^{2}\right)$ était alors égal à l'indice de Camacho-Sad-Lins Neto $C S_{m_{0}}(v, V)$ ([CS], [Li], [Su]) en $m_{0}$ relatif à $V$. Dans le cas des singularités qu'il a appelées "presque liouvilléenes" (qui comprennent en particulier les singularités simples: selles et selle-noeuds), Brunella a aussi donné une formule permettant de localiser le résidu au voisinage de certaines des séparatrices: celles qui constituent le pôle de la forme de Liouville.

Dans cet article, nous nous proposons d'abord de donner de telles formules de localisation dans le cas le plus général d'une singularité non dicritique $m_{0}$ : supposant le feuilletage défini par $v$ distinct de celui défini par $v_{f}$, nous montrons au paragraphe 2 que $P H(v)-\mu$ et $B B\left(v, c_{1}^{2}\right)$ ne dépendent, une fois choisie l'équation réduite $f=0$ de $V$, que du comportement local de $v$ dans un voisinage tubulaire de $\Sigma-\left\{m_{0}\right\}$, où l'ensemble $\Sigma$ (qui contient $V$ ) désigne l'ensemble des points en lesquels $v$ et $v_{f}$ sont colinéaires. Ces localisations dépendent donc aussi du choix de la fonction $f$, qui n'est définie qu'à unité près dans l'anneau des germes en $m_{0}$ de fonctions holomorphes (voir les remarques finales du paragraphe 2). Le paragraphe 3 est consacré à l'étude du comportement de ces indices par éclatement: nous y démontrons en particulier la réciproque du résultat précité de Brunella: si $G S V_{m_{0}}(v, V)=0$, la séparatrice est maximale, et le feuilletage est une courbe généralisée. Nous étudions des exemples au paragraphe 4 . Au paragraphe 5, nous donnons une interprétation de $G S V$ ainsi que de $P H-\mu$ en $K$-théorie.

Dans un article ultérieur, nous verrons ce qu'on peut dire en dimension supérieure.

Nous remercions les collègues avec qui nous avons eu d'utiles discussions, en particulier S. Azziz, M. Karoubi, A. Lins Neto, J. F. Mattei, M. Nicolau et M. Soarès.

\section{Théorème de localisation en dimension 2}

Soit donc $v=a \frac{\partial}{\partial x}+b \frac{\partial}{\partial y}$ comme dans l'introduction. (On notera $(v)$ le feuilletage défini par $v$ ). Soit $f=0$ une équation réduite de la séparatrice maximale $V$ passant par le point singulier isolé $m_{0}$ de $v$. Il existe alors une fonction holomorphe $C$ telle que

$$
<d f, v>=C f .
$$

Les champs de vecteurs $v$ et $v_{f}=f_{y}^{\prime} \frac{\partial}{\partial x}-f_{x}^{\prime} \frac{\partial}{\partial y}$ sont colinéaires sur l'ensemble $\Sigma$ 
des points où $C f=0$.

On supposera, dans toute la suite, les deux feuilletages distincts, c'est à dire $C$ non identiquement nulle. Nous appellerons "pseudo-séparatrice" toute branche de $C=0$ passant par $m_{0}$ et qui n'est pas une séparatrice. Soit $\mathcal{C}$ la réunion des pseudo-séparatrices. Notons $\left(V_{h}\right)_{h}$ la famille des branches irréductibles de $V$ en $m_{0}$, et $\left(\mathcal{C}_{h}\right)$ la famille des branches irréductibles de $\mathcal{C}$ en $m_{0}$. [On écrira en abrégé $h \in V$ ou $h \in \mathcal{C}$ pour préciser si l'on utilise des branches de l'un ou l'autre ensemble, tandis que $h \in \Sigma$ désignera indistinctement une branche $V_{h}$ ou $\mathcal{C}_{h}$.]

Une branche $B_{h}$ de $\Sigma$, d'équation réduite $f_{h}=0$, sera dite "simple" si aucune puissance $>1$ de $f_{h}$ ne divise $C f$, c'est à dire si:

- ou bien $h \in V$ et $f_{h}$ ne divise pas $C$,

- ou bien $h \in \mathcal{C}$, et $\left(f_{h}\right)^{2}$ ne divise pas $C$.

Soit $B_{h}$ une branche de $\Sigma$, et $U_{h}$ un voisinage tubulaire de $B_{h}-\left\{m_{0}\right\}$ dans $U$ (on supposera $U_{h} \cap U_{\ell}=\emptyset$ pour $k \neq \ell$. Quitte à effectuer un changement de coordonnées linéaires et à restreindre $U_{h}$, on peut toujours supposer que le champ de vecteurs $\frac{\partial}{\partial y}$ est transverse à $B_{h}-\left\{m_{0}\right\}$ et vérifie simultanément $v \wedge \frac{\partial}{\partial y} \neq 0$ et $v_{f} \wedge \frac{\partial}{\partial y} \neq 0$. Soit $X \mapsto(x=\varphi(X), y=\psi(X))$ une paramétrisation de Puiseux de $B_{h}$. On définit alors de nouvelles coordonnées locales $(X, Y)$ dans $U_{h}$ en posant:

$$
\left\{\begin{array}{l}
x=\varphi(X) \\
y=\psi(X)+Y
\end{array}\right.
$$

Remarquons d'une part que $B_{h}$ a pour équation $Y=0$, et d'autre part que ces coordonnées locales sont aussi valables en $m_{0}$ si $B_{h}$ est lisse.

Notons $A$ et $B$ (resp. $F$ et $G$ ) les composantes de $v$ (resp. $v_{f}$ ) relativement à la base $\left(\frac{\partial}{\partial X}, \frac{\partial}{\partial Y}\right)$ dans $U_{h}$. Posons $r=\frac{A}{F}$ : puisque $\frac{\partial}{\partial Y}$ (qui est égal à $\frac{\partial}{\partial y}$ ) est linéairement indépendant de $v$ et de $v_{f}, A$ et $F$ sont non nuls dans $U_{h}$.

Notons $J=J(v)$ la matrice jacobienne $\frac{D(A, B)}{D(X, Y)}$ donnant la partie linéaire de $v$, et posons $J_{f}=J\left(v_{f}\right)$.

On vérifie aisément les formules: $f_{Y}^{\prime}=\varphi^{\prime} F$ et $-f_{X}^{\prime}=\varphi^{\prime} G$, de sorte que $\operatorname{Tr}\left(J_{f}\right)=0$ si $B_{h}$ est lisse.

Soit maintenant $S^{3}$ une sphère de centre $m_{0}$ dans $U$, supposée transverse à chaque branche $B_{h}$. Notons $\Gamma_{h}$ la variété réelle $S^{3} \cap B_{h}$ (difféomorphe à un cercle), et notons $W_{h}$ un voisinage fermé de de $\Gamma_{h}$ dans $S^{3} \cap U_{h}$ : on peut toujours supposer $S^{3}$ définie de telle façon que les restrictions de la projection $(X, Y) \mapsto X$ à $W_{h}$ et $\partial W_{h}$ soient des fibrations au dessus de $\Gamma_{h}$.

Notons indistinctement $c_{I}$ l'un des deux polynômes de Chern $c_{2}$ ou $\left(c_{1}\right)^{2}$, et $B B\left(v, c_{I}\right)$ les résidus de Baum-Bott correspondants en $m_{0}$, le résidu $B B\left(v, c_{2}\right)$ n'étant autre que l'indice de Poincaré-Hopf $P H(v)$ en $m_{0}$. Pour toute matrice $M$ carrée d'ordre 2, on notera respectivement $c_{1}^{2}(M)$ et $c_{2}(M)$ le carré de la trace $\operatorname{Tr}^{2}(M)$ et le déterminant $\operatorname{dét}(M)$. 
Théorème 2.1. On a les deux formules:

$$
\begin{aligned}
& P H(v)-\mu=\sum_{h} R_{h}\left(v, c_{2}\right), \\
& \text { et } B B\left(v, c_{1}^{2}\right)=\sum_{h} R_{h}\left(v, c_{1}^{2}\right),
\end{aligned}
$$

où l'on a posé:

$$
\begin{aligned}
R_{h}\left(v, c_{I}\right) & =\frac{-1}{4 \pi^{2}} \int_{\partial W_{h}} \frac{c_{I}\left(J-r J_{f}\right)}{r C f} \varphi^{\prime} d X \wedge d Y \\
& =\frac{1}{2 i \pi} \int_{\Gamma_{h}}\left[\begin{array}{c}
c_{I}\left(J-r J_{f}\right) \\
r C f
\end{array}\right]_{X} \varphi^{\prime} d X,
\end{aligned}
$$

$\left[\begin{array}{c}c_{I}\left(J-r J_{f}\right) d Y \\ r C f\end{array}\right]_{X}$ désignant le résidu de Cauchy relatif à la variable $Y$ en $Y=$ 0, pour $X$ fixé. En particulier, sur une branche simple de $\Sigma$, on obtient:

$$
R_{h}\left(v, c_{I}\right)=\frac{1}{2 i \pi} \int_{\Gamma_{h}} \frac{c_{I}\left(J-r J_{f}\right)}{r D} \varphi^{\prime} d X
$$

où l'on a défini $D$ par l'égalité $C f=Y D\left(\right.$ avec $D \neq 0$ sur $\left.B_{h}-\left\{m_{0}\right\}\right)$.

Démonstration. Nous avons montré dans [L2], que pour deux feuilletages holomorphes distincts définis respectivement par les champs $v_{1}$ et $v_{2}$ admettant tous deux un même point singulier isolé $m_{0}$ de $\mathbb{C}^{n}$, la différence $B B\left(v_{2}, c_{I}\right)-B B\left(v_{1}, c_{I}\right)$ ne dépendait que du comportement de $v_{1}$ et $v_{2}$ dans un voisinage $U$ arbitrairement petit de l'ensemble $\Sigma_{0}$ des points de $U-\left\{m_{0}\right\}$ où $v_{1}$ et $v_{2}$ sont colinéaires. On rappelle qu'une connexion $\nabla$ sur $T M$ est dite "connexion de Bott" (ou "spéciale") relativement à un champ de vecteurs holomorphe $v^{\prime}$ partout non nul sur une variété holomorphe $M$, si

$$
\left\{\begin{aligned}
\nabla_{v^{\prime}} & =\left[v^{\prime}, .\right] \\
\nabla_{Z} Z^{\prime} & =0 \text { chaque fois que } Z^{\prime} \text { est holomorphe, et } Z \text { antiholomorphe. }
\end{aligned}\right.
$$

Notons alors:

$\nabla^{0}$ une connexion au dessus de $U_{h}-B_{h}$, spéciale à la fois pour $v$ et $v_{f}$,

$\nabla^{1}$ une connexion au dessus de $U_{h}$, spéciale à la fois pour $v$ et $\frac{\partial}{\partial Y}$,

$\nabla^{2}$ une connexion au dessus de $U_{h}$, spéciale à la fois pour $v_{f}$ et $\frac{\partial}{\partial Y}$.

De telles connexions existent toujours, et d'après la proposition 2 de [L2], dont nous reprenons les notations,

$$
\operatorname{Res}_{h}\left(v, c_{I}\right)=-\int_{\partial W_{h}} \Delta_{\omega_{0}, \omega_{1}, \omega_{2}}\left(c_{I}\right)
$$


où $\omega_{0}, \omega_{1}, \omega_{2}$ désignent les formes de connexion associées à $\nabla^{0}, \nabla^{1}, \nabla^{2}$ relativement à une même trivialisation de $T M$. (En effet, $\Delta_{\omega_{1}, \omega_{2}}\left(c_{I}\right)=0$, puisque $\nabla^{1}$ et $\nabla^{2}$ sont toutes deux spéciales pour le même champ de vecteurs $\left.\frac{\partial}{\partial Y}\right)$. Relativement à la trivialisation $\left(\frac{\partial}{\partial Y}, \frac{\partial}{\partial Y}\right)$ de $T M$, les formes de connexion sont respectivement données par

$$
\left\{\begin{array}{l}
\omega_{0}=\frac{1}{C f}\left[-d f J+\varphi^{\prime}(A d Y-B d X) J_{f}\right] \\
\omega_{1}=-\frac{d X}{A} J \\
\omega_{2}=-\frac{d X}{F} J_{f}
\end{array}\right.
$$

On en déduit:

$$
-\Delta_{\omega_{0}, \omega_{1}, \omega_{2}}\left(c_{I}\right)=\frac{1}{r C f} c_{I}\left(J-r J_{f}\right) \varphi^{\prime} d X \wedge d Y
$$

Notant en effet $c_{I}\left(\right.$. , . ) la forme polaire de la forme quadratique $c_{I}($. ), l'expression $\Delta_{\omega_{0}, \omega_{1}, \omega_{2}}\left(c_{I}\right)$ est toujours égale, d'après [B], à $c_{I}\left(\omega_{1}-\omega_{0}, \omega_{2}-\omega_{0}\right)$. [Si $\Delta$ désigne le simplexe $\{(t, u), t \geq 0, u \geq 0, t+u \leq 1\}$, $p_{2}$ la deuxième projection $\Delta \times\left(U_{h}-B_{h}\right) \rightarrow U_{h}-B_{h}$, et $\tilde{\omega}$ la connexion sur le fibré $\left(p_{2}\right)^{-1} T M$ égale à $t \omega_{1}+u \omega_{2}+(1-(t+u)) \omega_{0}$, la courbure $\tilde{\Omega}$ correspondante est en effet de la forme $d t \wedge\left(\omega_{1}-\omega_{0}\right)+d u \wedge\left(\omega_{2}-\omega_{0}\right)+K$, où $K$ désigne une expression sans $d t$ ni $d u$; l'image $c_{I}(\tilde{\Omega})$ de $c_{I}$ par le morphisme de Chern-Weil associé à $\tilde{\omega}$ est donc de la forme $d t \wedge d u c_{I}\left(\omega_{1}-\omega_{0}, \omega_{2}-\omega_{0}\right)$ modulo des termes sans $d t \wedge d u$, d'où l'expression de $\Delta_{\omega_{0}, \omega_{1}, \omega_{2}}\left(c_{I}\right)$ par intégration sur le simplexe $\Delta$.] On obtient donc:

$$
\begin{aligned}
-c_{I}\left(\omega_{1}-\omega_{0}, \omega_{2}-\omega_{0}\right) & =\frac{\varphi^{\prime}}{C f}\left[\frac{F}{A} c_{I}(J)-2 c_{I}\left(J, J_{f}\right)+\frac{A}{C} c_{I}\left(J_{f}\right)\right] d X \wedge d Y, \\
& =\frac{\varphi^{\prime} F}{A C f}\left[c_{I}(J)-2 \frac{A}{C} c_{I}\left(J, J_{f}\right)+\left(\frac{A}{C}\right)^{2} c_{I}\left(J_{f}\right)\right] d X \wedge d Y, \\
& =\frac{\varphi^{\prime}}{r C f}\left[c_{I}(J)-2 r c_{I}\left(J, J_{f}\right)+r^{2} c_{I}\left(J_{f}\right)\right] d X \wedge d Y, \\
& =\frac{1}{r C f} c_{I}\left(J-r J_{f}\right) \varphi^{\prime} d X \wedge d Y
\end{aligned}
$$

Remarques 2.2. 1) Dans le cas où $B_{h}$ est une séparatrice, $B$ et $G$ sont nuls sur $B_{h}$ (puisque $\frac{\partial}{\partial X}$ est tangent à $B_{h}$ ), et les hypothèses du théorème 1 de [L2], avec $n=2, v_{1}=v_{f}$ et $v_{2}=v$, sont vérifiées: la formule cherchée en est alors un cas particulier.

2) En dérivant par rapport à $Y$ l'identité $(-A G+B F) \varphi^{\prime} \equiv C f$, et en remarquant que $f, B$ et $G$ sont nuls sur une séparatrice, tandis que $A_{X}^{\prime}-r F_{X}^{\prime}=F r_{X}^{\prime}$ 
et $A_{Y}^{\prime}-r F_{Y}^{\prime}=F r_{Y}^{\prime}$, on voit que, sur une séparatrice,

$$
J-r J_{f}=\left(\begin{array}{cc}
F r_{X}^{\prime} & F r_{Y}^{\prime} \\
0 & C
\end{array}\right)
$$

Corollaire 2.3. Si $V_{h}$ est une branche simple de $V$, on obtient:

$$
R_{h}\left(v, c_{2}\right)=\frac{1}{2 i \pi} \int_{\Gamma_{h}} \frac{d r}{r} \text {, et } R_{h}\left(v, c_{1}^{2}\right)=\frac{1}{2 i \pi} \int_{\Gamma_{h}} \frac{\left(C+F r_{X}^{\prime}\right)^{2}}{A C} d X \text {. }
$$

Si, en outre, la séparatrice $V_{h}$ est lisse, $C+F r_{X}^{\prime}=A_{X}^{\prime}+B_{Y}^{\prime}$.

Démonstration. Sur $\Gamma_{h}, F$ est en effet égal à $\varphi^{\prime} f_{Y}^{\prime}$, tandis que $D=C f_{Y}^{\prime}, B=Y B_{Y}^{\prime}$, et $r$ est égal à $\frac{A}{f_{Y}^{\prime}}$. On en déduit: $\frac{c_{I}\left(J-r J_{f}\right)}{r D}=\frac{c_{I}\left(J-r J_{f}\right)}{A C}$. En outre, $c_{1}\left(J-r J_{f}\right)=$ $c_{1}(J)$ si $V_{h}$ est lisse.

Proposition 2.4. Posons, pour toute branche $V_{h}$ de la séparatrice $V$ :

$$
\left\{\begin{aligned}
G S V_{h}(v, V) & =\frac{1}{2 i \pi} \int_{\Gamma_{h}} \frac{d r}{r}, C S_{h}(v, V)=\frac{1}{2 i \pi} \int_{\Gamma_{h}} \frac{C}{A} d X \\
\operatorname{Var}_{h}(v, S) & =\frac{1}{2 i \pi} \int_{\Gamma_{h}} \frac{A_{X}^{\prime}+B_{Y}^{\prime}}{A} d X
\end{aligned}\right.
$$

On a alors:

$$
\left\{\begin{aligned}
G S V_{m_{0}}(v, V) & =\sum_{h \in S} G S V_{h}(v, V), C S_{m_{0}}(v, V)=\sum_{h \in S} C S_{h}(v, V), \\
\operatorname{Var}_{m_{0}}(v, V) & =\sum_{h \in S} \operatorname{Var}_{h}(v, V), \\
& \operatorname{Var}_{m_{0}}(v, V) \text { désignant la variation de Khanedani-Suwa }[\mathrm{KS}] .
\end{aligned}\right.
$$

Démonstration. D'après [Li], il existe des fonctions holomorphes $g$ et $k$, non nulles sur $V-\left\{m_{0}\right\}$ et dont les germes en $m_{0}$ sont premiers entre eux, ainsi qu'une 1-forme $\eta$ tels que

$$
g \omega=k d f+f \eta,
$$

$\omega$ désignant la forme $\omega=b d x-a d y$.

Notons $\Gamma$ la réunion des $\Gamma_{h}$ pour $h \in V$.

D'après [Br], $G S V_{m_{0}}(v, V)=\frac{1}{2 i \pi} \int_{\Gamma} \frac{d \frac{k}{g}}{\frac{k}{g}}$. Or il est clair que, sur $V_{h}, \frac{A}{F}=\frac{k}{g}$.

D'après [Li], [Su] $C S_{m_{0}}(v, V)=\frac{-1}{2 i \pi} \int_{\Gamma} \frac{\eta}{k}$. Or, sur $V_{h}, \frac{\eta}{k}=-\frac{C}{A} d X$, car les deux formes en question prennent la même valeur $C$ pour $f=0$ quand on les applique à $v$. 
Enfin, d'après [Br], [KS], $\operatorname{Var}_{m_{0}}(v, V)=G S V_{m_{0}}(v, V)+C S_{m_{0}}(v, V)$. Posons $f=Y \bar{f}$ sur $U_{h}$. On en déduit que $F=\bar{f}, f_{X}^{\prime}=Y \bar{f}_{X}^{\prime}$ et $B=Y B_{Y}^{\prime}$ sur $V_{h}$. A partir de l'identité $A f_{X}^{\prime}+B f_{Y}^{\prime}=c f$, on obtient d'autre part la relation $A \bar{f}_{X}^{\prime}=\bar{f}\left(C-B_{Y}^{\prime}\right)$. Il en résulte que $\frac{A_{X}^{\prime}+B_{Y}^{\prime}}{A}=\frac{\left(\frac{A}{F}\right)_{X}^{\prime}}{\frac{A}{F}}+\frac{C}{A}$ sur $V_{h}$, d'où:

$$
\operatorname{Var}_{h}(v, V)=G S V_{h}(v, V)+C S_{h}(v, V) .
$$

Remarques. D'après [Su], $C S_{m_{0}}(v, V)$ est en général distinct de $\sum_{h \in S} C S_{m_{0}}\left(v, V_{h}\right)$. Il ne faut donc pas confondre $C S_{h}(v, V)$ et $C S_{m_{0}}\left(v, V_{h}\right)$.

De façon analogue, $G S V_{h, m_{0}}(v, V) \neq G S V_{m_{0}}\left(v, V_{h}\right)$, car sur la branche $S_{h}$ d'équation réduite $f_{h}=0, v_{f_{h}}$ n'est pas la restriction de $v_{f}$.

Toutefois, (cf. [Br]), $\operatorname{Var}_{h}(v, V)=\operatorname{Var}_{m_{0}}\left(v, V_{h}\right)$.

Proposition 2.5. (i) Si $V_{h}$ est une branche simple de $V$, on obtient la formule:

$$
R_{h}\left(v, c_{2}\right)=G S V_{h}(v, V) .
$$

(ii) Si, en outre, $V_{h}$ est lisse, et vérifie la condition générique suivante: les restrictions à $V_{h}$ de $A, X\left(A_{X}^{\prime}+B_{Y}^{\prime}\right)$ et $X C$ sont toutes trois de même ordre d relativement à la variable $X$. Le nombre $C S_{h}(v, V)$ est alors nécessairement non nul, et

$$
R_{h}\left(v, c_{1}^{2}\right)=\frac{\left[\operatorname{Var}_{h}(v, V)\right]^{2}}{C S_{h}(v, V)} .
$$

En particulier, si $G S V_{h}(v, V)=0, R_{h}\left(v, c_{1}^{2}\right)=C S_{h}(v, V)$.

Démonstration. La partie (i) ne fait que reparaphraser l'une des formules du corollaire 2.3 .

Notons respectivement $\lambda X^{d}, \mu X^{d-1}$ et $\nu X^{d-1}$ les parties principales des restrictions de $A, A_{X}^{\prime}+B_{Y}^{\prime}$ et $C$ à $V_{h}$. On a alors:

$$
\begin{aligned}
R_{h}\left(v, c_{1}^{2}\right) & =\frac{1}{2 i \pi} \int_{\Gamma_{h}} \frac{\left(A_{X}^{\prime}+B_{Y}^{\prime}\right)^{2}}{A C} d X, \\
& =\frac{\mu^{2}}{\lambda \nu} .
\end{aligned}
$$

De façon analogue, $C S_{h}(v, V)=\frac{\nu}{\lambda}$, et $\operatorname{Var}_{h, m_{0}}(v, V)=\frac{\mu}{\lambda}$. La partie (ii) de la proposition résulte immédiatement de ces trois égalités.

Remarques concernant le choix de l'équation de $V$. 1) Il faut noter que $V$ ne dépend que du feuilletage défini par $v$, tandis que le feuilletage défini par $v_{f}$, 
ainsi que $C$ et les pseudo-séparatrices dépendent du choix de l'équation réduite $f=0$ de $V$ : si $f$ est remplacé par $u f$, où $u$ désigne une unité (fonction holomorphe ne s'anulant pas sur $U), v_{f}$ est remplacé par $v_{u f}=u v_{f}+f v_{u}$, et $\langle d(u f), v\rangle=$ $\left(C+\frac{v \cdot u}{u}\right) u f$.

2) Si $C\left(m_{0}\right) \neq 0$, il n'existe pas de pseudo-séparatrice en $m_{0}$, et ceci quelque soit le choix de $f$.

3) Si $C\left(m_{0}\right)=0$, un nombre fini d'éclatements permet de faire disparaître les pseudo-séparatrices, puisque l'on finira toujours par obtenir que les transformées strictes de celles ci ne passent plus par les points singuliers du feuilletage relevé. En particulier, si, après réduction des singularités, il reste des pseudo-séparatrices, celles ci passent nécessairement pas des points selle ayant des valeurs propres $\lambda$ et $\mu$ opposées: on pourra alors éclater encore une fois de tels points selle.

4) Il est toujours possible de choisir l'équation réduite $f=0$ de $V$, de façon que toutes les séparatrices soient simples. En effet, les points $(\lambda, \mu)$ de $\mathbb{C}^{2}$ tels que $\lambda a+\mu b$ ne soit divisible par aucun des facteurs premiers $f_{h}$ de $f$ forment un ouvert dense. Il suffit alors de remplacer $f$ par $\exp (\lambda x+\mu y) f$ pour un tel couple $(\lambda, \mu)$, et chaque séparatrice est devenue branche simple.

5) Si le feuilletage défini par $v$ est la restriction d'un feuilletage (à singularités isolées) défini sur une surface holomorphe compacte $M$ admettant une séparatrice compacte maximale $V$, l'équation de $V$ est bien définie, à constante multiplicative près, au voisinage de tout point singulier du feuilletage.

Par exemple sur l'espace projectif $\mathbb{P}_{\mathbb{C}}(2)$, dont nous noterons $[u, v, w]$ les coordonnées homogènes, le feuilletage $\mathcal{F}$ défini par le flot

$$
\left.\left(([u, v, w]) \mapsto\left[e^{t \lambda} u, e^{t \mu} v, e^{t \nu} w\right]\right)\right)_{t \in \mathbb{C}}
$$

à partir de 3 nombres complexes $(\lambda, \mu, \nu)$ deux à deux distincts, admet une séparatrice compacte maximale, d' équation réduite $u v w=0$. Le feuilletage $\left(u v-\lambda w^{2}\right)_{\lambda \in \mathbb{C}}$ est alors défini globalement et de façon naturelle. Au voisinage du point $[0,0,1]$, il coïncide avec $\left(v_{f}\right)$ pour $f(x, y)=x y\left(\operatorname{avec} x=\frac{u}{w}, y=\frac{v}{w}\right)$, $f=0$ étant une équation particulière de la séparatrice maximale locale de $\mathcal{F}$. Tel ne serait pas le cas, par exemple, si l'on prenait $x y(1+x)=0$ comme équation réduite de cette séparatrice maximale locale.

Malheureusement, l'exemple de Jouanolou ([J]) montre qu'une telle séparatrice globale peut ne pas exister, et que c'est même ce qui se passe génériquement (cf. Lins Neto [Li]), alors que localement il en existe toujours d'après Camacho-Sad $([\mathrm{CS}])$.

\section{Comportement des indices par éclatements}

Soit $d$ l'ordre du champ de vecteurs en $m_{0}$, et $k$ l'ordre d'une séparatrice $V$, non nécessairement maximale pour le moment, d'équation réduite $f=0$. Soit $\left(x^{\prime}, t\right)$ 
les coordonnées locales dans la variété obtenue en éclatant le point $m_{0}$, quand on écrit la projection $\left(x^{\prime}, t\right) \mapsto\left(x=x^{\prime}, y=x^{\prime} t\right)$. On note $\hat{a}, \hat{b}$ et $\hat{f}$ les fonctions "éclatées-divisées"

$$
\hat{a}\left(x^{\prime}, t\right)=\frac{1}{x^{\prime d}} a\left(x^{\prime}, t x^{\prime}\right), \hat{b}\left(x^{\prime}, t\right)=\frac{1}{x^{\prime}} b\left(x^{\prime}, t x^{\prime}\right), \hat{f}\left(x^{\prime}, t\right)=\frac{1}{x^{\prime k}} f\left(x^{\prime}, t x^{\prime}\right) .
$$

Puisque la singularité $m_{0}$ est supposée non dicritique, le feuilletage relevé est défini par le champ $\tilde{v}=x^{\prime} \hat{a} \frac{\partial}{\partial x^{\prime}}+(\hat{b}-t \hat{a}) \frac{\partial}{\partial t}$ dans le domaine de la carte $\left(x^{\prime}, t\right)$. Une équation réduite de la réunion $\tilde{V}=\hat{V} \cup \mathbb{P}_{\mathbb{C}}(1)$ de la transformée stricte $\hat{V}$ de $V$ et du diviseur exceptionnel, s'écrit alors: $x^{\prime} \hat{f}=0$. [C'est une séparatrice pour $\tilde{v}$, maximale si $V$ est maximale pour $v$.] On posera: $\tilde{a}=x^{\prime} \hat{a}$ et $\tilde{b}=\hat{b}-t \hat{a}$, et $\tilde{f}=x^{\prime} \hat{f}$.

On peut toujours supposer les coordonnées $(x, y)$ choisies de façon que le feuilletage relevé ait toutes ses singularités $\tilde{m}_{i}=\left(0, t_{i}\right)$ dans le domaine de la carte $\left(x^{\prime}, t\right)$. Les nombres $t_{i}$ sont les racines du polynôme $\tilde{b}(0, t)$. Soit $\beta_{i}$ leur ordre de multiplicité: $\sum_{i} \beta_{i}=d+1$. Les racines de $\hat{f}(o, t)$ sont certains des nombres $t_{i}$ ci-dessus (tous, si la séparatrice $V$ est maximale), avec un ordre de multiplicité $\alpha_{i}$. [On posera $\alpha_{i}=0$ si $t_{i}$ n'est pas racine de $\hat{f}(o, t)$.]

Avec les notations précédentes, on vérifie aisément:

$$
v_{\tilde{f}}=\widetilde{\left(v_{f}\right)}+(k-1) \hat{f} \frac{\partial}{\partial t}
$$

Une branche irréductible $V_{h}$ de $V$ admet une paramétrisation de Puiseux de la forme $\left(x=z^{p_{h}}, y=c z^{q_{h}}+\ldots\right)$ où $p_{h}$ et $q_{h}$ sont des entiers premiers entre eux. La notation $h \in i$ signifiera que la transformée stricte $\hat{V}_{h}$ de $V_{h}$ passe par $\tilde{m}_{i}$. Posons $W_{i}=\cup_{h \in i} V_{h}$. Si $V$ est maximale, $\tilde{W}_{i}$ est une séparatrice maximale pour $\tilde{v}$ admettant encore $\tilde{f}=0$ comme équation réduite.

Rappelons le résultat suivant:

Théorème 3.1 ([CLS]). La singularité $m_{0}$ étant supposée non dicritique, les relations suivantes sont vérifiées:

(i) $\beta_{i}-\alpha_{i} \geq 0$ pour tout $i$, et $d-k+1 \geq 0$.

(ii) $\beta_{i}-\alpha_{i}=0$ pour tout $i$, et $d-k+1=0$ pour une courbe généralisée, si la séparatrice est maximale.

Remarque. J. F. Mattei et E. Salem ([MS]) ont démontré plus précisément que $d-k+1$ est nul pour la séparatrice maximale d'une singularité non dicritique, lorsque les espaces tangents qui correspondent à la valeur propre 0 en les points selle-noeuds de la réduction des singularités sont tangents à des transformées strictes, et non à une composante du diviseur exceptionnel.

Brunella a démontré $([\mathrm{Br}])$ que $G S V_{m_{0}}(v, V)$ est toujours positif ou nul pour une singularité non dicritique. On obtient plus précisément le 
Théorème 3.2. (i) La formule suivante est vérifiée:

$$
G S V_{h}(v, V)=G S V_{h}(\tilde{v}, \tilde{V})+p_{h}(d-k+1),
$$

où $G S V_{h}(\tilde{v}, \tilde{V})$ désigne la contribution de $\hat{V}_{h}$ (et non de $\tilde{V}_{h}$ ) à $G S V_{\tilde{m}_{i}}(\tilde{v}, \tilde{V})$ pour $h \in i$. [En particulier, $G S V_{h}(v, V) \geq G S V_{h}(\tilde{v}, \tilde{V})$, l'égalité ne se produisant que si $d-k+1=0$.]

(ii) $G S V_{m_{0}}(v, V)=\sum_{i} G S V_{\tilde{m}_{i}}(\tilde{v}, \tilde{V})+\left[\left(\sum_{h \in V} p_{h}\right)-1\right](d-k+1)$. [En particulier, $G S V_{m_{0}}(v, V) \geq \sum_{i} G S V_{\tilde{m}_{i}}(\tilde{v}, \tilde{V})$, l'égalité ne se produisant que si $d-k+1=$ 0.]

(iii) $G S V_{h}(v, V) \geq 0$.

Démonstration. Posons $v=r_{h} v_{f}$ sur $V_{h}$. Sur $\tilde{V}_{h}$, on en déduit $\tilde{v}=\tilde{r}_{h} v_{\tilde{f}}$, avec $\tilde{r}_{h}=\frac{r_{h}}{x^{\prime}-k+1}$, d'où $\frac{d r_{h}}{r_{h}}=\frac{d \tilde{r}_{h}}{\tilde{r}_{h}}+(d-k+1) \frac{d x^{\prime}}{x^{\prime}}$. Utilisant la paramétrisation de Puiseux, on obtient la première partie du théorème.

La contribution du diviseur exceptionnel à $G_{S} V_{\tilde{m}_{i}}(\tilde{v}, \tilde{V})$ est égale à $\beta_{i}-\alpha_{i}$ puisque $\tilde{v}=\frac{\tilde{b}}{-\hat{f}} v_{\tilde{f}}$ pour $x^{\prime}=0$. (En effet, pour $\left.x^{\prime}=0, \widehat{f}_{x}^{\prime}+t \widehat{f}_{y}^{\prime}=k \widehat{f}\right)$. Compte tenu de (i), et de ce que $\sum_{i}\left(\beta_{i}-\alpha_{i}\right)=d-k+1$, on obtient la formule (ii).

La formule (i) montre que $G S V_{h}(v, V)$ ne peut que diminuer par éclatement. Comme il est positif ou nul pour une singularité réduite, on en déduit (iii).

D'après $[\mathrm{Br}], G S V_{m_{0}}(v, V)=0$ dans le cas d'une courbe généralisée si la séparatrice est maximale. En fait la réciproque est vraie:

Théorème 3.3. Les conditions suivantes sont équivalentes:

(i) $G S V_{m_{0}}(v, V)=0$,

(ii) $G S V_{h}(v, V)=0$ pour tout $h \in V$,

(iii) Le feuilletage défini par $v$ est une courbe généralisée en $m_{0}$, et $V$ est la séparatrice maximale.

Démonstration. L'inégalité (iii) du théorème 3.2 implique l'équivalence des parties (i) et (ii) du théorème.

Supposons maintenant $G S V_{m_{0}}(v, V)=0$. Si $m_{0}$ est déja réduite, la partie (ii) du théorème est évidente. Sinon, $G S V_{\tilde{m}_{i}}(\tilde{v}, \tilde{V})=0$ pour tout $i$ et $d-k+1=0$ d'après la partie (ii) du théorème 3.2. Par récurrence sur le nombre d'éclatements nécessaire à la réduction des singularités, on voit qu'il ne peut apparaître de sellenoeud où $G S V$ serait strictement positif. On a donc une courbe généralisée. En outre, si la séparatrice n'était pas maximale, $d-k+1$ serait strictement positif.

Le fait que (iii) implique (i) dans le théorème est aussi un corollaire immédiat du théorème 3.2 . 
Mentionnons pour mémoire le

Théorème 3.4 ([CLS]). (i) La formule suivante est vérifiée:

$$
P H_{m_{0}}(v)-\mu_{m_{0}}(f)=\sum_{i}\left[P H_{\tilde{m}_{i}}(\tilde{v})-\mu_{\tilde{m}_{i}}(\tilde{f})\right]+(d-k+1)(d+k-2),
$$

où $\mu_{m_{0}}(f)$ (resp. $\left.\mu_{\tilde{m}_{i}}(\tilde{f})\right)$ désigne le nombre de Milnor de la séparatrice $f=0$ (resp. $\tilde{f}=0)$ en $m_{0}$ (resp. $\left.\tilde{m}_{i}\right)$.

En particulier, $P H_{m_{0}}(v)-\mu_{m_{0}}(f) \geq \sum_{i}\left[P H_{\tilde{m}_{i}}(\tilde{v})-\mu_{\tilde{m}_{i}}(\tilde{f})\right]$, l'égalité ne se produisant que si $d-k+1=0$.

(ii) On a toujours l'inégalité

$$
P H_{m_{0}}(v)-\mu_{m_{0}}(f) \geq 0
$$

l'égalité $n$ 'ayant lieu que pour une courbe généralisée, la séparatrice $f=0$ de $v$ étant alors automatiquement maximale.

\section{Exemples}

1) Exemple $a=x^{2}, b=y(\lambda x+y)$, en fonction du paramètre complexe $\lambda$, que l'on supposera égal à 1 ou non rationnel. [Si $\lambda$ était un rationnel différent de 1 , l'origine serait une singularité dicritique; tous les calculs d'indice resteraient cependant valables; on ne pourrait simplement plus parler de courbe généralisée ou de séparatrice maximale.]

Puisque $x b-y a=x y[(\lambda-1) x+y]$, la séparatrice maximale a pour équation $x b-y a=0$ si $\lambda \notin \mathbb{Q}$, et $x y=0$ si $\lambda=1$. En outre, si $\lambda \neq 1, C=\operatorname{Tr} J=$ $(\lambda+2) x+2 y$. Notons respectivement $S_{1}, S_{2}, S_{3}$ et $\mathcal{C}_{4}$ les droites d'équations $y=0, x=0,(\lambda-1) x+y=0$, et $(\lambda+2) x+2 y=0$. On distinguera alors trois cas:

$\lambda \notin \mathbb{Q}$ : on a une courbe généralisée, avec 3 séparatrices simples $S_{1}, S_{2}, S_{3}$ et une pseudo-séparatrice simple $\mathcal{C}_{4}$. Les invariants sont donnés par le tableau:

$$
\begin{array}{cccccc} 
& S_{1} & S_{2} & S_{3} & \mathcal{C}_{4} & \text { Total } \\
G S V_{h}(v, V) & 0 & 0 & 0 & & G S V(v, V)=0 \\
C S_{h}(v, V) & \lambda+2 & 2 & 4-\lambda & & C S(v, V)=8 \\
R_{h}\left(v, c_{1}^{2}\right) & \lambda+2 & 2 & 4-\lambda & 0 & B B\left(v, c_{1}^{2}\right)=8 \\
R_{h}\left(v, c_{2}\right) & 0 & 0 & 0 & 0 & P H(v)-\mu=0
\end{array}
$$


En fait, il est aisé de calculer directement $P H(v)=\operatorname{dim}\left[\mathcal{O}(x, y) /\left(x^{2}, y(\lambda x+y)\right)\right]=$ 4 , et $\mu=\operatorname{dim}[\mathcal{O}(x, y) /(y(y+2(\lambda-1) x), x(2 y+(\lambda-1) x))]=4$.

$\underline{\lambda=1}$ : ce n'est pas une courbe généralisée, $d-k+1=1$, il y a deux séparatrices simples $S_{1}$ et $S_{2}$, et une pseudo-séparatrice simple $\mathcal{C}$; les invariants sont décrits par le tableau suivant:

$$
\begin{array}{ccccc} 
& S_{1} & S_{2} & \mathcal{C} & \text { Total } \\
G S V_{h}(v, V) & 1 & 1 & & G S V(v, V)=2 \\
C S_{h}(v, V) & 2 & 1 & & C S(v, V)=3 \\
R_{h}\left(v, c_{1}^{2}\right) & \frac{9}{2} & 4 & -\frac{1}{2} & B B\left(v, c_{1}^{2}\right)=8 \\
R_{h}\left(v, c_{2}\right) & 1 & 1 & 1 & P H(v)-\mu=3
\end{array}
$$

En fait, il est aisé de calculer directement $P H(v)=\operatorname{dim} \frac{\mathcal{O}(x, y)}{\left(x^{2}, y^{2}-x y\right)}=4$, et $\mu=$ $\operatorname{dim} \frac{\mathcal{O}(x, y)}{(x, y)}=1$.

2) Exemple d'un point selle avec valeurs propres opposées. L'intérêt de cet exemple est double:

- d'une part, il s'agit d'un point selle, avec une pseudo-séparatrice dont la contribution à $B B\left(v, c_{1}^{2}\right)$ est non nulle. Il n'est donc pas tout à fait exact, dans ce cas, d'affirmer que $B B\left(v, c_{1}^{2}\right)$ se "localise" au voisinage des seules séparatrices. Cependant, il reste vrai que $B B\left(v, c_{1}^{2}\right)=C S(v, V)$ (en l'occurence 0 ).

- d'autre part on peut voir, sur cet exemple, comment la contribution de chaque séparatrice ou pseudo-séparatrice dépend en général du choix de l'équation réduite de la séparatrice maximale, et peut même en dépendre de façon discontinue.

Soient en effet $\lambda$ et $t$ deux paramètres dans $C$ (on supposera $\lambda \neq 0$ ), $v$ le champ de vecteurs

$$
\left\{\begin{array}{l}
a=x(1+\lambda x+y) \\
b=-y
\end{array}\right.
$$

$f(x, y)=e^{-\lambda x+y+t y^{2}} x y$.

On a alors: $C=-\left(\lambda^{2} x^{2}+\lambda x y+2 t y^{2}\right)$. La séparatrice maximale est formée des deux branches simples $y=0$ et $x=0$.

Posons $\xi^{2}=1-8 t$, de sorte que $-4 C=(2 \lambda x+y)^{2}-\xi^{2} y^{2}$ : selon que $\xi$ est nul ou non, on a une pseudo-séparatrice double d'équation $2 \lambda x+y=0$ ou deux pseudo-séparatrices simples d'équations $2 \lambda x+(1-\epsilon \xi) y=0$, avec $\epsilon= \pm 1$.

Pour la branche $y=0$, on obtient: $R_{h}\left(v, c_{1}^{2}\right)=3$. Pour la branche $x=0$, $R_{h}\left(v, c_{1}^{2}\right)$ est égal à 3 ou à $\frac{1}{2 t}$, selon que $t$ est nul ou non.

Si $\xi \neq 0$, on obtient: $R_{h}\left(v, c_{1}^{2}\right)=\frac{2 \epsilon \xi}{\epsilon \xi-1}$ pour la pseudo-séparatrice $2 \lambda x+$ $(1-\epsilon \xi) y=0$. 
Si $\xi=0$, on obtient: $R_{h}\left(v, c_{1}^{2}\right)=0$ pour la pseudo-séparatrice $2 \lambda x+y=0$.

3) Exemple d'un selle-noeud. Donnons nous un entier $p \geq 1$, un nombre complexe non nul $\lambda$, et prenons le champ de vecteurs, correspondant à la forme normale du selle-noeud, défini par: $a=x^{p+1}, b=y\left(1+\lambda x^{p}\right)$, avec $f(x, y)=x y$, et $C=1+(\lambda+1) x^{p}$. Puisque $C$ ne s'annule pas à l'origine, il n'y a pas de pseudo-séparatrice. Dans cet exemple, l'hypothèse de généricité de 2.5 (ii) n'est pas vérifiée, et si $\lambda \neq p^{2}-2 p-1$, on a $R_{h}\left(v, c_{1}^{2}\right) \cdot C S_{h} \neq\left(\operatorname{Var}_{h}\right)^{2}$ pour la branche $y=0$.

Les invariants sont donnés par le tableau:

$$
S_{1}(y=0) \quad S_{2}(x=0) \quad \text { Total }
$$

$\begin{array}{cccc}G S V_{h}(v, V) & p & 0 & G S V(v, V)=p \\ C S_{h}(v, V) & \lambda+1 & 1 & C S(v, V)=\lambda+2 \\ R_{h}\left(v, c_{1}^{2}\right) & \lambda+1+2 p & 1 & B B\left(v, c_{1}^{2}\right)=\lambda+2+2 p \\ R_{h}\left(v, c_{2}\right) & p & 0 & P H(v)-\mu=p\end{array}$

Il est aisé de calculer directement $P H(v)=p+1$, et $\mu=1$.

\section{Localisation de $P H-\mu$ et expression de GSV en K-théorie}

\section{1) Rappels sur l'homomorphisme de Chern-Weil en K-théorie relative}

Soit $X$ un espace topologique ayant le type d'homotopie d'un $C W$ complexe, et $Y$ un sous espace topologique ayant le type d'homotopie d'un $C W$ sous complexe. Rappelons d'abord qu'un élémént de la K-théorie complexe relative $K(X, Y)=$ $\tilde{K}(X / Y)$ peut se définir par la donnée d'un triple $(P, Q, \phi)$, où $P$ et $Q$ désignent des fibrés vectoriels complexes au dessus de $X$, et $\phi$ un isomorphisme $\phi:\left.\left.P\right|_{Y} \cong Q\right|_{Y}$ entre les restrictions de ces fibrés à $Y$. On notera $[P, Q, \phi]$ l'élément de $K(X, Y)$ ainsi défini, qui ne dépend en particulier que de la classe d' isotopie de $\phi$ (mais nous renvoyons à $[\mathrm{A}]$ pour préciser la relation d'équivalence entre triples exprimant qu'ils définissent le même élément de $K(X, Y))$. L'image de $[P, Q, \phi]$ par l'application naturelle $K(X, Y) \rightarrow K(X)$ est égale à $[Q-P]$. Rappelons aussi les relations $-[P, Q, \phi]=\left[Q, P, \phi^{-1}\right]$, et $[P, Q, \phi]+[Q, R, \psi]=[P, R, \psi \circ \phi]$.

Si $X$ est une variété différentiable, $Y$ un ouvert de $X$, et $Y_{1}$ un autre ouvert tel que $X=Y \cup Y_{1}$, rappelons ([L1]) que la cohomologie $H^{*}(X, Y)$ à coefficients complexes peut se définir par le complexe de Mayer-Viétoris relatif

$$
M V^{*}(X, Y)=\Omega_{D R}^{*}\left(Y_{1}\right) \oplus \Omega_{D R}^{*-1}\left(Y \cap Y_{1}\right),
$$


muni de la différentielle $D:\left(u_{1}, v\right) \rightarrow\left(d u_{1},-d v+u_{1}\right), \Omega_{D R}^{*}$ désignant l'algèbre des formes différentielles et $d$ la différentielle de de-Rham. (Le théorème d'excision correspond au fait que la cohomologie de ce complexe ne dépend pas du choix de $Y_{1}$.)

Soit $[P, Q, \phi] \in K(X, Y)$ et supposons $P, Q$ et $\phi$ différentiables. Donnons nous deux connexions $\nabla_{1}^{P}$ sur la restriction $\left.P\right|_{Y_{1}}$ de $P$ à $Y_{1}$, et $\nabla_{1}^{Q}$ sur $\left.Q\right|_{Y_{1}}$. Notant $c(\nabla)=1+c_{1}(\nabla)+c_{2}(\nabla)+\cdots$ le cocycle représentant la classe totale de Chern d'un fibré par l'homomorphisme de Chern-Weil associé à une connexion $\nabla$ sur ce fibré, la classe totale de Chern de $[P, Q, \phi]$ est donnée, d'après [LS] (2-13), par la classe de cohomologie du cocycle

$$
\left(\frac{c\left(\nabla_{1}^{P}\right)}{c\left(\nabla_{1}^{Q}\right)}, \frac{c\left(\phi\left(\nabla_{1}^{P}\right), \nabla_{1}^{Q}\right)}{c\left(\nabla_{1}^{Q}\right)}\right) \in M V^{*}(X, Y)
$$

où $c\left(\nabla, \nabla^{\prime}\right)$ représente l'opérateur différence de Bott tel que $d c\left(\nabla, \nabla^{\prime}\right)=c\left(\nabla^{\prime}\right)-$ $c(\nabla)$. En particulier, si les connexions $\nabla_{1}^{P}$ et $\nabla_{1}^{Q}$ sont triviales, le cocycle précédent a une partie homogène de degré $2 j(j>0)$ égale à $\left(0, c_{j}\left(\phi\left(\nabla_{1}^{P}\right), \nabla_{1}^{Q}\right)\right)$.

\section{2) Localisation de $P H-\mu$ en K-théorie}

Rappelons ([BB1]) qu'un feuilletage $\mathcal{F}$ (de dimension complexe 1) sur une surface holomorphe $M$ (dimension complexe 2) est défini par la donnée d'un fibré holomorphe en droites complexes $\mathcal{L}$, de base $M$, et d'un morphisme holomorphe $\ell: \mathcal{L} \rightarrow T M$ de fibrés vectoriels, l'ensemble singulier de $\mathcal{F}$ étant l'ensemble des points de $M$ où $\ell$ n'est pas injectif. Mais $\mathcal{F}$, étant aussi de codimension (complexe) 1 , peut encore être défini par la donnée d'un fibré holomorphe en droites complexes $\mathcal{N}$, de base $M$, et d'un morphisme holomorphe $\omega: T M \rightarrow \mathcal{N}$ de fibrés vectoriels, l'ensemble singulier de $\mathcal{F}$ étant alors égal à l'ensemble des points de $M$ où $\omega$ n'est pas surjectif. Soit $S$ une partie de $M$ contenant les points singuliers de $\mathcal{F}$ et qu'on supposera compacte. La suite $0 \rightarrow \mathcal{L} \stackrel{\ell}{\rightarrow} T M \stackrel{\omega}{\rightarrow} \mathcal{N} \rightarrow 0$ est exacte au dessus de $M-S$. La donnée d'une scission (différentiable ou continue) de cette suite exacte permet de définir un isomorphisme $\phi(\mathcal{F}): \mathcal{L} \oplus \mathcal{N} \stackrel{\phi}{\rightarrow} T M$ au dessus de $M-S$, dont la classe d'isotopie ne dépend pas du choix de la scission: soit $K P H(\mathcal{F})=[\mathcal{L} \oplus \mathcal{N}, T M, \phi(\mathcal{F})]$ l'élément ainsi défini dans $K(M, M-S)$. Si $\left(S_{\alpha}\right)_{\alpha}$ désigne la famille des composantes connexes de $S, K(M, M-S)$ est égal à $\oplus_{\alpha} K\left(M, M-S_{\alpha}\right)$, et l'on notera $K P H_{\alpha}(\mathcal{F})$ la composante de $K P H(\mathcal{F})$ sur $K\left(M, M-S_{\alpha}\right)$. Supposons en particulier que $S_{\alpha}$ soit un point singulier isolé $\left\{m_{0}\right\}$ avec $\mathcal{F}=(v)$ au voisinage de $m_{0}$; on notera $K P H_{\alpha}(\mathcal{F})=K P H_{m_{0}}(v)$. D'après le théorème d'excision en K-théorie, on peut restreindre alors $M$ à un voisinage contractile $U$ de $m_{0}$ dans $M$, arbitrairement petit, admettant des coordonnées holomorphes $(x, y)$, et au dessus duquel tous les fibrés sont triviaux. Notant $\theta_{2}(U)$ le fibré trivial de rang 2 au dessus de $U$, et posant $v=a \frac{\partial}{\partial x}+b \frac{\partial}{\partial y}$, $K P H_{m_{0}}(v)$ est alors égal à $\left[\theta_{2}(U), T(U), \phi_{v}\right]$, où $\phi_{v}$ désigne la trivialisation de 
$T\left(U-m_{0}\right)$ définie par les deux vecteurs $v$ et $\hat{v}=\frac{\bar{b}}{\delta} \frac{\partial}{\partial x}-\frac{\bar{a}}{\delta} \frac{\partial}{\partial y}$. La première classe de Chern de $K P H_{m_{0}}(v)$ est nécessairement nulle, puisque $H^{2}\left(M, M-m_{0}\right)=0$.

Lemme 5.1. La deuxième classe de Chern $c_{2}$ de $K P H_{m_{0}}(v)$ est égale à $P H_{m_{0}}(v)$ dans $H^{4}\left(M, M-m_{0}\right) \cong \mathbb{Z}$.

Ce résultat est probablement bien connu et doit faire partie du "folklore", mais comme nous ne l'avons pas trouvé dans la littérature, nous allons en donner rapidement la démonstration.

D'après les rappels faits en début de paragraphe, $c_{2}\left[\theta_{2}(U), T(U), \phi(v)\right]$ est égal à la classe de cohomologie du cocycle $\left(0, c_{2}\left(\phi_{v}\left(\nabla_{1}^{0} \oplus \nabla_{1}^{0}\right), \nabla_{2}^{0}\right)\right)$ dans $M V^{4}\left(U, U-m_{0}\right)$, où $\nabla_{1}^{0}$ désigne la connexion triviale naturelle sur le fibré trivial de rang $1 \theta_{1}(U)$, et $\nabla_{2}^{0}$ la connexion triviale sur $T U$ associée à la trivialisation $\left(\frac{\partial}{\partial x}, \frac{\partial}{\partial y}\right)$. Si $D$ est un disque disque de dimension 4 dans $U$ autour de $m_{0}$, l'évaluation du cocycle précédent sur le cycle relatif $[D, \partial D]$ est égale à $\left.-\int_{\partial D} c_{2}\left(\phi_{v}\left(\nabla_{1}^{0} \oplus \nabla_{1}^{0}\right), \nabla_{2}^{0}\right)\right)$ lorsque la sphère $\partial D$ est orientée par la normale sortante. Puisque la connexion $\phi_{v}\left(\nabla_{1}^{0} \oplus \nabla_{1}^{0}\right)$ est $v$ triviale, cette intégrale est égale à $P H_{m_{0}}(v)$.

Soient $v$ et $v^{\prime}$ deux champs de vecteurs sur le voisinage $U$ de $m_{0}$, admettant $m_{0}$ comme seule singularité éventuelle dans $U$ (en particulier, $v^{\prime}$ pourra être égal à $v_{f}$ ). Soit $\Sigma$ l'ensemble des points de $U$ en lesquels $v$ et $v^{\prime}$ sont colinéaires. Soit $D$ un disque autour de $m_{0}$ comme précédemment.

Théorème 5.2. (i) La différence $K P H_{m_{0}}\left(v^{\prime}\right)-K P H_{m_{0}}(v)$ dans $K\left(U, U-m_{0}\right)$ admet un relèvement naturel

$$
K P H_{0}\left(v, v^{\prime}\right) \in K^{-1}\left(U-m_{0}\right)
$$

par l'application $K^{-1}\left(U-m_{0}\right) \stackrel{\partial}{\rightarrow} K\left(U, U-m_{0}\right)$ de la suite exacte du couple $\left(U, U-m_{0}\right)$.

(ii) L'élément $K P H_{0}\left(v, v^{\prime}\right)$ admet un relèvement naturel

$$
K P H\left(v, v^{\prime}\right) \in K^{-1}\left(U-m_{0}, U-\Sigma\right)
$$

par l'application $K^{-1}\left(U-m_{0}, U-\Sigma\right) \rightarrow K^{-1}\left(U-m_{0}\right)$ de la suite exacte du couple $\left(U-m_{0}, U-\Sigma\right)$.

Démonstration. D'après les rappels faits en début de paragraphe, $K P H_{m_{0}}\left(v^{\prime}\right)-$ $K P H_{m_{0}}(v)$ est égal à $\left[\theta_{2}(U), \theta_{2}(U), \phi_{v}^{-1} \circ \phi_{v^{\prime}}\right]$. Puisque $\phi_{v}^{-1} \circ \phi_{v^{\prime}}$ peut encore se lire comme une application de $U-m_{0}$ dans $G L(2, \mathbb{C})$, elle définit (cf. [A]) un fibré de rang 2 sur la suspension de $U-m_{0}$, donc un élément naturel de $K^{-1}\left(U-m_{0}\right)$ dont 
l'image par $K^{-1}\left(U-m_{0}\right) \stackrel{\partial}{\rightarrow} K\left(U, U-m_{0}\right)$ est égale à $K P H_{m_{0}}\left(v^{\prime}\right)-K P H_{m_{0}}(v)$, d'où la partie (i) du théorème.

On vérifie: $\phi_{v}^{-1} \circ \phi_{v^{\prime}}=\left(\begin{array}{cc}\frac{\alpha}{\delta} & \frac{-\bar{\beta}}{\delta \delta^{\prime}} \\ \beta & \frac{\bar{\alpha}}{\delta^{\prime}}\end{array}\right)$, avec $\alpha=\bar{a} a^{\prime}+\bar{b} b^{\prime}$ et $\beta=b a^{\prime}-a b^{\prime}$.

Pour $t \in[0,1]$, posons: $g_{t}=\left(\begin{array}{cc}\frac{t+(1-t) \alpha}{t+(1-t) \delta} & \frac{-(1-t) \bar{\beta}}{t+(1-t) \delta \delta^{\prime}} \\ (1-t) \beta & \frac{t+(1-t) \bar{\alpha}}{t+(1-t) \delta^{\prime}}\end{array}\right)$. Cette matrice a un déterminant qui est toujours positif ou nul, mais qui est strictement positif si $\beta \neq 0$, c'est à dire sur $U-\Sigma$ : l'application $g_{t}$ est donc une isotopie entre l'application constante égale à l'identité et $\phi_{v}^{-1} \circ \phi_{v^{\prime}}: U-\Sigma \rightarrow G L(2, \mathbb{C})$. C'est dire que la restriction de $K P H_{0}\left(v, v^{\prime}\right)$ à $U-\Sigma$ est nulle, et que $K P H_{0}\left(v, v^{\prime}\right)$ admet un relèvement à $K^{-1}\left(U-m_{0}, U-\Sigma\right)$, lequel est canonique puisque l'isotopie $g_{t}$ sur $U-\Sigma$ a été définie de façon naturelle, d'où la partie (ii) du théorème.

\section{3) Expression de GSV en K-théorie}

Une séparatrice de $\mathcal{F}$ est un sous ensemble analytique $V$ de $M$, tel que $V_{0}=$ $V-S_{V}$ soit une feuille de $\mathcal{F}$, où l'on a posé $S_{V}=S \cap V$. Le fibré normal à $V_{0}$ admet deux extensions, de nature différente, à tout $V$ : ce sont respectivement le fibré en droites $E$ associé au diviseur définissant $V$, et la restriction à $V$ du fibré normal virtuel $\mathcal{Q}(\mathcal{F})=T M-\mathcal{L}$ de Baum-Bott. Plus précisément, $V$ est défini comme l'ensemble des zéros d'une section holomorphe $V$ d'un fibré holomorphe $E \rightarrow M^{\prime}$ défini au dessus d'un voisinage $M^{\prime}$ de $V$ dans $M, V$ étant génériquement transverse à la section 0 de $E$. Notant $\nabla$ une connexion arbitraire sur $E$, rappelons ([LS]) que la restriction à $V$ du morphisme $\nabla s: T M^{\prime} \rightarrow E$ ne dépend pas du choix de $\nabla$, et que sa restriction à $V_{0}$ est surjective et a pour noyau $T V_{0}$, permettant d'identifier canoniquement $\left.E\right|_{V_{0}}$ au fibré normal de $V_{0}$ dans $M$. Dire que $V$ est une séparatrice de $\mathcal{F}$ signifie alors que la suite de fibrés vectoriels

$$
\left.\left.\left.0 \rightarrow \mathcal{L}\right|_{V} \stackrel{\ell}{\rightarrow} T M\right|_{V} \stackrel{\nabla s}{\rightarrow} E\right|_{V} \rightarrow 0
$$

est exacte au dessus de $V-S_{V}$ : une scission de cette suite exacte définit par conséquent un isomorphisme $\left.\left.[\mathcal{L} \oplus E]\right|_{V-S_{V}} \rightarrow T M\right|_{V-S_{V}}$, d'où un élément

$$
\operatorname{KGSV}(\mathcal{F}, V) \in K\left(V, V-S_{V}\right) .
$$

Supposons en particulier que $m_{0}$ soit un point isolé de $S$ et que $V$ passe par $m_{0}$. On notera alors $K G S V_{m_{0}}(\mathcal{F}, V)$ ou $K G S V_{m_{0}}(v, V)$ l'image de $K G S V(\mathcal{F}, V)$ dans $K\left(V, V-m_{0}\right)$ induite par l'inclusion de $m_{0}$ dans $S_{V}$. Soit $U$ un voisinage de $m_{0}$ dans $M$ ne contenant pas d'autre point de $S$. Posons $U_{V}=U \cap V$. Le théorème d'excision en $K$-théorie permet alors d'affirmer que l'application naturelle $K\left(V, V-m_{0}\right) \rightarrow K\left(U_{V}, U_{V}-m_{0}\right)$ est un isomorphisme. 
Si l'on note d'autre part $\left(V_{h}\right)_{h=1, \cdots, N}$ la famille des branches localement irréductibles de $V$ en $m_{0}$, on a le lemme suivant:

Lemme 5.3. Les inclusions $\left(V_{h}, V_{h}-m_{0}\right) \rightarrow\left(V, V-m_{0}\right)$ définissent une famille de projecteurs $K\left(V, V-m_{0}\right) \rightarrow K\left(V_{h}, V_{h}-m_{0}\right)$ permettant d'identifier $K\left(V, V-m_{0}\right)$ à la somme directe $\oplus_{h=1}^{N} K\left(V_{h}, V_{h}-m_{0}\right)$, laquelle est elle même naturellement isomorphe $\grave{a} \mathbb{Z}^{N}$.

Démonstration. Chaque branche $V_{h}$ admettant un paramétrage de Puiseux, on peut choisir le voisinage $U$ de $m_{0}$ dans $M$ de telle sorte que chaque intersection $U \cap V_{h}$ contienne un disque $D_{h}$ de dimension 2. L'espace quotient $V_{h} /\left(V_{h}-m_{0}\right)$ a même type d'homotopie que $D_{h} / \partial D_{h}$ qui est homéomorphe à une sphère $S_{h}^{2}$ de dimension 2, munie de deux points de base: d'une part le centre qui a pour image $m_{0}$ par le paramétrage de Puiseux (et qui sera encore noté $m_{0}$ par abus de notation), et d'autre part la classe d' équivalence $m_{h}$ de $\partial D_{h}$. L'espace quotient $V /\left(V-m_{0}\right)$ a donc même type d'homotopie que le quotient du bouquet $\bigvee_{h} S_{h}^{2}$ par le sous espace fini $\left\{m_{1}, \cdots, m_{h}, \cdots m_{N},\right\}$. La suite exacte associée à cette cofibration en $K$-théorie montre alors que le morphisme naturel $\tilde{K}\left(V /\left(V-m_{0}\right)\right) \rightarrow$ $\tilde{K}\left(\bigvee_{h} S_{h}^{2}\right)=\mathbb{Z}^{N}$ induit par la cofibration est en fait un isomorphisme, chaque facteur $\mathbb{Z}$ correspondant à une composante $\tilde{K}\left(V_{h} /\left(V_{h}-m_{0}\right)\right)$.

On notera $K G S V_{h}(\mathcal{F}, V)$ la composante de $K G S V_{m_{0}}(\mathcal{F}, V) \in K\left(V, V-m_{0}\right)$ sur $K\left(V_{h}, V_{h}-m_{0}\right)$.

Attention à ne pas confondre $K G S V_{h}(\mathcal{F}, V)$ avec $K G S V\left(\mathcal{F}, V_{h}\right)$ !

Théorème 5.4. La donnée de $G S V_{m_{0}}(\mathcal{F}, V)$ équivaut à celle de $K G S V_{m_{0}}(v, V)$. Plus précisément, l'isomorphisme

$$
c_{1}: K\left(V_{h}, V_{h}-m_{0}\right) \rightarrow H^{2}\left(V_{h}, V_{h}-m_{0}\right) \cong \mathbb{Z}
$$

défini par la première classe de Chern applique $K G S V_{h}(v, V)$ sur $G S V_{h}(\mathcal{F}, V)$.

Remarque. La formule $c_{1}(K G S V(v, V))=G S V(\mathcal{F}, V)$ est contenue implicitement dans le théorème 4.5 de $[\mathrm{LS}]$.

Démonstration. Soit $U_{0}$ un voisinage tubulaire de $V_{0}$ dans $M$, et soit $U_{1}$ un sous voisinage de $m_{0}$ dans $U$ tel que $U_{0} \cup U_{1}$ admette une rétraction par déformations sur $V$ (de tels choix de $U_{0}$ et $U$ sont toujours possibles). La cohomologie relative $H^{*}\left(V, V-m_{0}\right)$ est alors donnée par celle du complexe $M V^{*}\left(U_{0} \cup U_{1}, U_{0}\right)$. Soit $\nabla$ la connexion triviale sur $T M$ au dessus de $U_{1}$ définie par $\nabla \frac{\partial}{\partial x}=0$ et $\nabla \frac{\partial}{\partial y}=0$. Soit $\nabla^{\prime}$ la connexion triviale sur $\left.T M\right|_{V_{0}}$ au voisinage de $m_{0}$ définie par $\nabla^{\prime} v=0$ et $\nabla^{\prime} w=0$, où $v=a \frac{\partial}{\partial x}+b \frac{\partial}{\partial y}$ et $w=\alpha \frac{\partial}{\partial x}+\beta \frac{\partial}{\partial y}$ avec $\alpha=\frac{-r \bar{b}}{a \bar{a}+b \bar{b}}, \beta=\frac{r \bar{a}}{a \bar{a}+b \bar{b}}$, et $r$ tel que $v=r v_{f}$ au dessus de $V_{0}$. [Ces composantes $\alpha$ et $\beta$ sont définies de façon que 
$w$ soit à la fois orthogonal à $v$ pour la métrique hermitienne canonique sur $C^{2}$ et vérifie: $\langle d f, w\rangle \equiv 1$.]

D'après les rappels faits en début de section, $c_{1}\left(K G S V_{h}(v, V)\right)$ est donnée par le cocycle $\left(0, c_{1}\left(\nabla, \nabla^{\prime}\right)\right)$ dans $M V^{2}\left(\left(U_{0} \cup U_{1}, U_{0}\right)\right.$, et son évaluation sur le cycle relatif $\left(D_{h}, \partial D_{h}\right)$ par l'intégrale $\int_{\Gamma_{h}} c_{1}\left(\nabla, \nabla^{\prime}\right)$ où $\Gamma_{h}=\partial D_{h}$.

Relativement à la trivialisation $\left(\frac{\partial}{\partial x}, \frac{\partial}{\partial y}\right)$ de $T M$ au voisinage de $m_{0}, \nabla$ a pour forme de connexion $\eta$ la matrice 0 , tandis que $\nabla^{\prime}$ a pour forme de connexion la matrice $\left(\begin{array}{ll}p & q \\ u & s\end{array}\right)$, avec $a p+b q+d a=0, a u+b s+d b=0, \alpha p+\beta q+d \alpha=0$, et $\alpha u+\beta s+d \beta=0$. On en déduit: $p=\frac{1}{r}(b d \alpha-\beta d a), s=\frac{1}{r}(\alpha d b-a d \beta)$, et $p+s=\frac{-d r}{r}$, puisque $r=a \beta-b \alpha$. Or $c_{1}\left(\nabla, \nabla^{\prime}\right)=-(p+s)$. Il en résulte que l'évaluation de $c_{1}\left(K G S V_{h}(v, V)\right)$ sur $\Gamma_{h}$ est égale à $\int_{\Gamma_{h}} \frac{d r}{r}$, c'est à dire à $G S V_{h}(v, V)$.

Des théorèmes 3.3, 5.2 et 5.4, résulte immédiatement le

Corollaire 5.5. Les trois propriétés suivantes d'un feuilletage $\mathcal{F}$ admettant en un point $m_{0}$ une singularité non dicritique sont équivalentes:

(i) $\mathcal{F}$ est une courbe généralisée,

(ii) $K G S V(v, V)=0$ dans $K\left(V, V-m_{0}\right)$,

(iii) $K P H\left(v, v_{f}\right)=0$ dans $K\left(M-m_{0}, M-\Sigma\right)$.

\section{Références}

[A] M. F. Atiyah, K-theory, Notes by D. W. Anderson, Harvard University, Cambridge, Mass. 1964.

[B] R. Bott, Lectures on characteristic classes and foliations, Lectures on Algebraic and Differential Topology, Lecture Notes in Mathematics 279, Springer-Verlag, 1972, 1-94.

[BB1] P. Baum and R. Bott, On the zeroes of meromorphic vector-fields, Essays on Topology and Related Topics, Memoires dédiés à Georges de Rham, Springer-Verlag, New York, Heidelberg, Berlin, 1970, 29-47.

[BB2] P. Baum and R. Bott, Singularities of holomorphic foliations, J. Differential Geom. 7 (1972), 279-342.

[Br] M. Brunella, Some remarks on indices of holomorphic vector fields, Publ. Mat. 41 No 2 (1997), 527-544.

[CS] C. Camacho and P. Sad, Invariant varieties through singularities of holomorphic vector fields, Ann. of Math. 115 (1982), 579-595.

[CLS] C. Camacho, A. Lins Neto and P. Sad, Topological invariants and equidesingularization for holomorphic vector fields, J. Differential Geometry 20 (1984), 143-174.

[GSV] X. Gomez-Mont, J. Seade and A. Verjovski, The index of a holomorphic flow with an isolated singularity, Math. Ann. 291 (1991), 737-751.

[KS] B. Khanedani and T. Suwa, First variations of holomorphic forms and some applications, Hokkaido Math. J. 26 (1997), 323-335.

[J] J. C. Jouanolou, Equations de Pfaff algébriques, Vol. 708, Lecture Notes in Math., SpringerVerlag, 1979 
[L1] D. Lehmann, Classes caractéristiques résiduelles, Differential Geometry and its applications, Proc. Conf. Aug. 27 - Sept. 2, 1989, Brno, Czechosl., World Scientific, Singapore, 1990, 85-108.

[L2] D. Lehmann, Residues of higher order and holomorphic vector fields, Annals of Global Analysis and Geometry 12 (1994), 109-122.

[LS] D. Lehmann and T. Suwa, Generalization of variations and Baum-Bott residues for holomorphic foliations on singular varieties, International J. of Maths. 10-3 (1999), 367-384.

[Li] A. Lins Neto, Algebraic solutions of polynomial differential equations and foliations in dimension two, Holomorphic Dynamics, Mexico 1986, Lecture Notes in Mathematics 1345, Springer-Verlag, New York, Heidelberg, Berlin, 1988, 192-232.

[MS] J. F. Mattei et E. Salem, Classification topologique et analytique des feuilletages génériques de $\left(C^{2}, 0\right)$, en préparation.

[Se] A. Seidenberg, Reduction of singularities of the differentiable equation $A d Y=B d X$, Amer. J. Math. 90 (1968), 248-269.

[Su] T. Suwa, Indices of holomorphic vector fields relative to invariant curves on surfaces, Proc. of the Am. Math. Soc. 123 (1995), 2989-2997.

Vincent Cavalier

Département des Sciences Mathématiques

Université de Montpellier II

Place Eugène Bataillon

F-34095 Montpellier Cedex 5

France

e-mail: cavalier@darboux.math.univ-montp2.fr e-mail: lehmann@darboux.math.univ-montp2.fr

Daniel Lehmann

Département des Sciences Mathématiques

Université de Montpellier II

Place Eugène Bataillon

F-34095 Montpellier Cedex 5

France

(Received: August 25, 2000) 